\title{
Association of metabolic syndrome with various anthropometric and atherogenic parameters in the Kazakh population in China
}

\author{
Xiaocui Chen ${ }^{1 \dagger}$, Chunhui He ${ }^{1,2+}$, Yitong Ma ${ }^{1,2^{*}}$, Yining Yang ${ }^{1,2}$, Fen Liu' ${ }^{1}$, Xiang Ma ${ }^{1,2}$, Xiaomei Li ${ }^{1,2}$, Xiang Xie \\ and Bangdang Chen ${ }^{1^{*}}$
}

\begin{abstract}
Background: This study aimed to evaluate the association of the metabolic syndrome (MetS) with various anthropometric and atherogenic parameters in adult Kazakh population in China.

Methods: Four thousand ninety-four Kazakhs were recruited since 2007 to 2010. MetS and its components were confirmed according to IDF criteria. Area under the curve (AUC) of each variable was compared. Sensitivity (Sen), specificity (Spe), shortest distance in receiver's operating characteristic curve (ROC) and cutoff of each variable to diagnose MetS were calculated.

Results: $28.6 \%$ of men and $31.0 \%$ of women had MetS in the Kazakh population. In men, WHtR had the highest AUC value 0.821, followed by BMI (0.801), TG/HDL-C (0.792), WHR (0.776) and BAI (0.666). In women, WHtR also had the highest AUC value (0.835), following by BMI (0.789), WHR (0.778), TG/HDL-C (0.778) and BAI (0.751). WHtR had the shortest ROC distance that was 0.37 and the optimal cutoff was 0.55 in men. In women, WHtR also had the shortest ROC distance of 0.35 and the optimal cutoff was 0.54 .
\end{abstract}

Conclusion: WHtR is the best predictor of MetS in both Kazakh men and women according to the IDF criteria.

Keywords: Metabolic syndrome, Body mass index, Waist to height ratio, Waist to hip ratio, Body adiposity index, TG/HDL-C

\section{Background}

Metabolic syndrome (MetS) is a cluster of metabolic abnormalities characterized as central obesity, raised triglyceride (TG) level, reduced high density lipoprotein cholesterol (HDL-C), raised blood pressure, raised fasting plasma glucose, or previously diagnosed type 2 diabetes [1]. It will exacerbate the progression of cardiovascular disease (CVD) if left untreated [2]. In recent years, many studies have focused on optimal obese and atherogenic indices to predict metabolic syndrome [3-5]. Nevertheless,

\footnotetext{
*Correspondence: myt-xj@163.com; chenbangdang@126.com

${ }^{\dagger}$ Equal contributors

${ }^{1}$ Xinjiang Key Laboratory of Cardiovascular Disease, Clinical Medical Research Institute, First Affiliated Hospital of Xinjiang Medical University, Urumqi 830054, China

Full list of author information is available at the end of the article
}

controversy still remains over which anthropometric parameters convey the highest risk of metabolic syndrome.

Body mass index (BMI) has been used to assess body fat for almost 200 years. Waist to height ratio (WHtR) has recently been regarded as the best screening tool for detection of cardiometabolic risk factors, especially in Asian populations [6-9]. Some studies proposed waist circumference (WC) or waist to hip ratio (WHR) [10-12] as the best anthropometric parameters to predict the metabolic syndrome, whereas others advocated their combined use [13, 14]. Although BMI, WHtR, WC and WHR are simple and convenient anthropometric parameters for epidemiological studies, their validity in measuring adiposity has been questioned since they cannot differentiate between fat and lean mass [15]. One study had reported that body adiposity index (BAI) could be used to reflect 
\%body fat for adult men and women of different ethnicities without numerical correction [3]. A study based on Chinese postmenopausal women showed that TG/HDL-C is the best to predict the presence of metabolic syndrome [5].

This cross-sectional study aimed to identify the association of metabolic syndrome with various anthropometric and atherogenic parameters (BMI, WHtR, WHR, BAI and TG/HDL-C) in Kazakh population in Xinjiang Uighur Autonomous Region, northwest of China.

\section{Results}

\section{Baseline characteristics of the study participants}

Table 1 summarized the baseline characteristics of the participants with or without MetS in Kazakh men and women. In men, the participants with MetS tended to have significantly elder age and higher height, weight, waist circumference, hip circumference, SBP, DBP, fasting glucose, total cholesterol, triglycerides, BMI, WHR, WHtR, BAI, TG/HDL-C when compared with the participants without MetS $(P<0.001)$. The participants without MetS tended to have significantly higher HDL-C and LDL-C compared with the participants with MetS $(P<0.001)$. In women, the parameters were higher in participants with MetS than those in participants without MetS except for that the height, HDL-C and LDL-C were lower in participants with MetS compared with that in participants without MetS.

\section{Prevalence of MetS and its components}

Prevalence of MetS and its individual components in Kazakh men and women were shown in Fig. 1. After age adjustment, $28.6 \%$ of men had the MetS while $31.0 \%$ of women had the MetS, the prevalence of MetS in women and men were not significantly different $(P=0.091)$. As for the individual components of MetS, the prevalence of central obesity, raised $\mathrm{TG}$, raised $\mathrm{BP}$ and raised FPG in women was significantly lower than that in men $(P<0.001)$. However, the prevalence of reduced HDL-C was not significantly different among men and women $(P=0.063)$.

\section{Association of each anthropometric and atherogenic parameter with MetS by ROC}

Table 2 presented the AUC of each variable for the presence of MetS in Kazakh men and women. In men, according to the respective ROC curves, the WHtR had the highest AUC value ( $\mathrm{AUC}=0.821$ ), it was followed by BMI $(\mathrm{AUC}=0.801)$, TG/HDL-C $(\mathrm{AUC}=0.792)$, WHR $(\mathrm{AUC}=0.776)$ and BAI $(\mathrm{AUC}=0.666)$. In women, according to the respective ROC curves, the WHtR also had the highest AUC value (AUC =0.835), it was followed by BMI (AUC $=0.789)$, WHR (AUC $=0.778)$, TG/HDL-C $(\mathrm{AUC}=0.778)$ and BAI $(\mathrm{AUC}=0.751)$. Figure 2 showed ROC curves for each variable to predict the presence of MetS in Kazakh men and women.

Table 1 Characteristics of the participants with or without MetS in Kazakh men and women

\begin{tabular}{|c|c|c|c|c|c|c|}
\hline & \multicolumn{3}{|l|}{ Men $(n=1999)$} & \multicolumn{3}{|l|}{ Women $(n=2095)$} \\
\hline & With MetS $(n=571)$ & Without MetS $(n=1428)$ & $\overline{P \text { value }}$ & With MetS $(n=649)$ & Without MetS $(n=1446)$ & $P$ value \\
\hline Age (year) & $50.61 \pm 11.67$ & $48.70 \pm 12.14$ & $0.001^{*}$ & $53.54 \pm 10.20$ & $45.57 \pm 10.92$ & $<0.001^{*}$ \\
\hline Waist circumference $(\mathrm{cm})$ & $101.77 \pm 8.91$ & $87.53 \pm 11.26$ & $<0.001^{*}$ & $95.27 \pm 9.91$ & $80.21 \pm 12.02$ & $<0.001^{*}$ \\
\hline Hip circumference (cm) & $105.03 \pm 7.77$ & $97.98 \pm 8.57$ & $<0.001^{*}$ & $104.61 \pm 10.00$ & $96.40 \pm 9.20$ & $<0.001^{*}$ \\
\hline $\mathrm{SBP}(\mathrm{mmHg})$ & $152.56 \pm 22.87$ & $137.37 \pm 22.51$ & $<0.001^{*}$ & $154.57 \pm 25.01$ & $131.62 \pm 23.69$ & $<0.001^{*}$ \\
\hline $\mathrm{DBP}(\mathrm{mmHg})$ & $97.93 \pm 17.85$ & $87.18 \pm 18.20$ & $<0.001^{*}$ & $96.87 \pm 20.27$ & $81.52 \pm 18.49$ & $<0.001^{*}$ \\
\hline Fasting glucose (mmol/L) & $5.91 \pm 2.26$ & $4.94 \pm 1.05$ & $<0.001^{*}$ & $5.56 \pm 2.00$ & $4.80 \pm 0.97$ & $<0.001^{*}$ \\
\hline Total cholesterol (mmol/L) & $5.19 \pm 1.25$ & $4.65 \pm 1.15$ & $<0.001^{*}$ & $5.18 \pm 1.09$ & $4.54 \pm 1.08$ & $<0.001^{*}$ \\
\hline $\mathrm{LDL}-\mathrm{C}(\mathrm{mmol} / \mathrm{L})$ & $2.77 \pm 0.87$ & $2.94 \pm 0.93$ & $<0.001^{*}$ & $2.78 \pm 0.92$ & $2.98 \pm 0.94$ & $<0.001^{*}$ \\
\hline $\mathrm{HDL}-\mathrm{C}(\mathrm{mmol} / \mathrm{L})$ & $1.10 \pm 0.41$ & $1.36 \pm 0.43$ & $<0.001^{*}$ & $1.10 \pm 0.34$ & $1.38 \pm 0.41$ & $<0.001^{*}$ \\
\hline Triglycerides (mmol/L) & $1.95 \pm 1.55$ & $1.08 \pm 0.78$ & $<0.001^{*}$ & $1.45 \pm 0.82$ & $0.92 \pm 0.45$ & $<0.001^{*}$ \\
\hline $\mathrm{BMI}\left(\mathrm{kg} / \mathrm{m}^{2}\right)$ & $29.99 \pm 3.69$ & $25.71 \pm 3.79$ & $<0.001^{*}$ & $29.68 \pm 4.86$ & $24.60 \pm 4.50$ & $<0.001^{*}$ \\
\hline WHR & $0.97 \pm 0.07$ & $0.89 \pm 0.09$ & $<0.001^{*}$ & $0.91 \pm 0.08$ & $0.83 \pm 0.08$ & $<0.001^{*}$ \\
\hline $\mathrm{WHtR}$ & $0.60 \pm 0.05$ & $0.52 \pm 0.07$ & $<0.001^{*}$ & $0.61 \pm 0.07$ & $0.51 \pm 0.08$ & $<0.001^{*}$ \\
\hline $\mathrm{BAl}$ & $29.45 \pm 4.11$ & $27.14 \pm 4.22$ & $<0.001^{*}$ & $35.40 \pm 5.71$ & $30.88 \pm 4.88$ & $<0.001^{*}$ \\
\hline TG/HDL-C & $1.99 \pm 2.32$ & $0.92 \pm 1.13$ & $<0.001^{*}$ & $1.45 \pm 0.99$ & $0.77 \pm 0.65$ & $<0.001^{*}$ \\
\hline
\end{tabular}

MetS metabolic syndrome, SBP systolic blood pressure, $D B P$ diastolic blood pressure, $L D L-C$ low-density lipoprotein cholesterol, $T G$ triglycerides, $H D L-C$ high-density lipoprotein cholesterol, $B M I$ body mass index, WHR waist to hip ratio, WHtR waist to height ratio, $B A I$ body adiposity index

${ }^{*} P$ value $<0.05$ compared to With MetS 


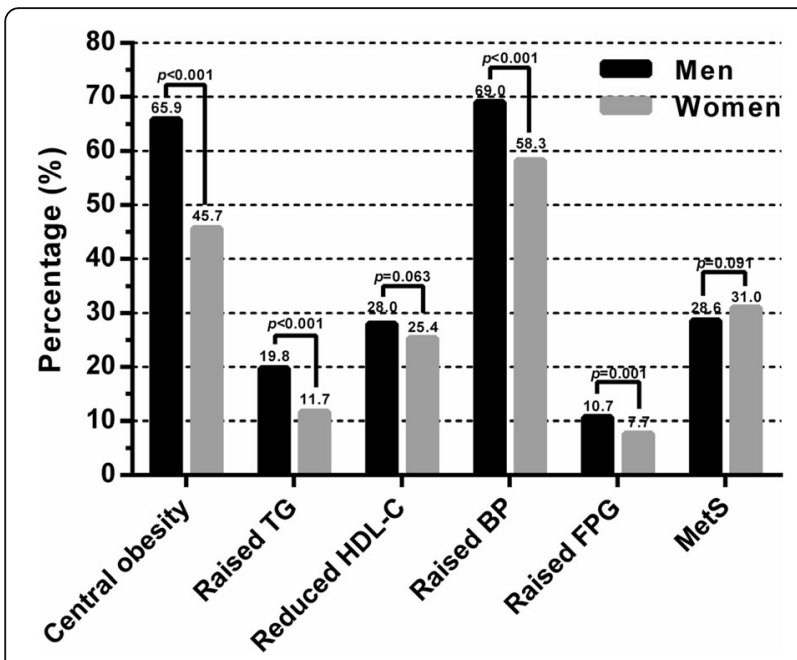

Fig. 1 Age adjusted prevalence of MetS and its individual components in Kazakh men and women. MetS, metabolic syndrome; TG, triglycerides; HDL-C, high-density lipoprotein cholesterol; BP, blood pressure; FPG, fasting plasma glucose

\section{Cutoff of each anthropometric and atherogenic parameter in MetS with ROC}

Table 3 showed the sensitivity, specificity, distance in the ROC and cutoffs of each variable for the presence of MetS in Kazakh men and women. In men, among all five anthropometric and atherogenic parameters, WHtR had the shortest distance of 0.37 in ROC (Sen $=81.09 \%$, Spe $=68.50 \%$ ), the cutoff of WHtR was 0.55. Following WHtR, the association of each parameter to MetS was followed by BMI, TG/ HDL-C, WHR and BAI according to the distance in ROC. In women, among all five anthropometric and atherogenic parameters, WHtR also had the shortest distance of 0.35 in ROC (Sen $=84.59 \%$, Spe $=68.97 \%$ ), the cutoff of WHtR was 0.54. Following WHtR, the association was followed by BMI, TG/HDL-C, WHR, and BAI according to the distance in ROC.

Table 2 Area under the curves of each variable for the presence of MetS in Kazakh men and women

\begin{tabular}{|c|c|c|}
\hline Varibles & AUC $(95 \% \mathrm{Cl})$ in Men & AUC $(95 \% \mathrm{Cl})$ in Women \\
\hline $\mathrm{BMI}$ & $0.801(0.781,0.821)$ & $0.789(0.769,0.809)$ \\
\hline WHR & $0.776(0.755,0.796)$ & $0.778(0.758,0.798)$ \\
\hline $\mathrm{WHtR}$ & $0.821(0.803,0.839)$ & $0.835(0.818,0.852)$ \\
\hline $\mathrm{BAl}$ & $0.666(0.640,0.692)$ & $0.751(0.728,0.773)$ \\
\hline TG/HDL-C & $0.792(0.769,0.815)$ & $0.778(0.756,0.800)$ \\
\hline
\end{tabular}

MetS metabolic syndrome, AUC Area under the curves, $B M I$ body mass index, $W H R$ waist to hip ratio, $W H t R$ waist to height ratio, $B A l$ body adiposity index, TG triglycerides, $H D L-C$ high-density lipoprotein cholesterol

The bolded data represent the highest AUC (areas under the curve) of ROC curve with the WHtR

\section{Discussion}

The total Kazakh population was 1.46 million in 2010 in China, $99.57 \%$ of the total Kazakh population live in Xinjiang Uighur Autonomous Region that is located in the center of Asia. This study provides the populationbased data on the prevalence of MetS in Kazakh population aged over 35 years. To our knowledge, this is the first large scale, population based, cross-sectional survey to estimate the prevalence of MetS in Kazakh population in Xinjiang, China. In this present study, $28.6 \%$ of the men had MetS while $31.0 \%$ of the women had MetS according to IDF criteria. The prevalence of MetS between men and women was not significantly different $(P=$ 0.091), which was different from other studies both in China and other countries [16-18].

Age-standardized prevalence of MetS was $28.8 \%$ according to IDF criteria based on Chinese postmenopausal women in Guangdong province [5]. The agestandardized prevalence of MetS was $28.6 \%$ in women based on elderly Chinese adults in Jiangsu province [19]. Our study investigated the Kazakh population within the same age range. We found that the prevalence of MetS in Kazakh women was higher than that in Chinese women in Guangdong province [4, 5]. One study including a total of 15,838 Chinese individuals showed that $31.1 \%$ of female participants had $\mathrm{BMI} \geq 25,37.8 \%$ of female participants had the $\mathrm{WC}>80 \mathrm{~cm}$ [20]. The reason for the higher prevalence of MetS and obesity in Kazakh women was still unclear. The eating and living habit may play an important role, the Kazakh population consumed more pasta, meat and milk products than the Han population did. The majority of Kazakh women tended to consume food containing a large amount of calories and lipids for a long time after they gave birth, it may greatly contribute to the high prevalence of MetS and obesity in Kazakh women.

In men, the WHtR had the highest AUC value of 0.821, it was followed by BMI, TG/HDL-C, WHR and BAI. Similarly, among all five anthropometric and atherogenic parameters, WHtR had the shortest distance of 0.37 in ROC. The association of WHtR to MetS was followed by BMI, TG/HDL-C, WHR and BAI according to the distance in ROC. Our results were consist with the study by Zhang et al. [4], they reported WHtR was best predicting indicator of MetS in Chinese men in Gunagdong province with the AUC of 0.69. The reason why WHtR may be a better predictor of metabolic risk was that it took height into account. An association between short height and risk of coronary heart disease was previously reported [21]. Henriksson et al. found that body height was negatively related to serum cholesterol and non-HDL cholesterol in middle-aged men, which was independent of BMI and WHR [22]. Thus, it may be important to take height into consideration. 

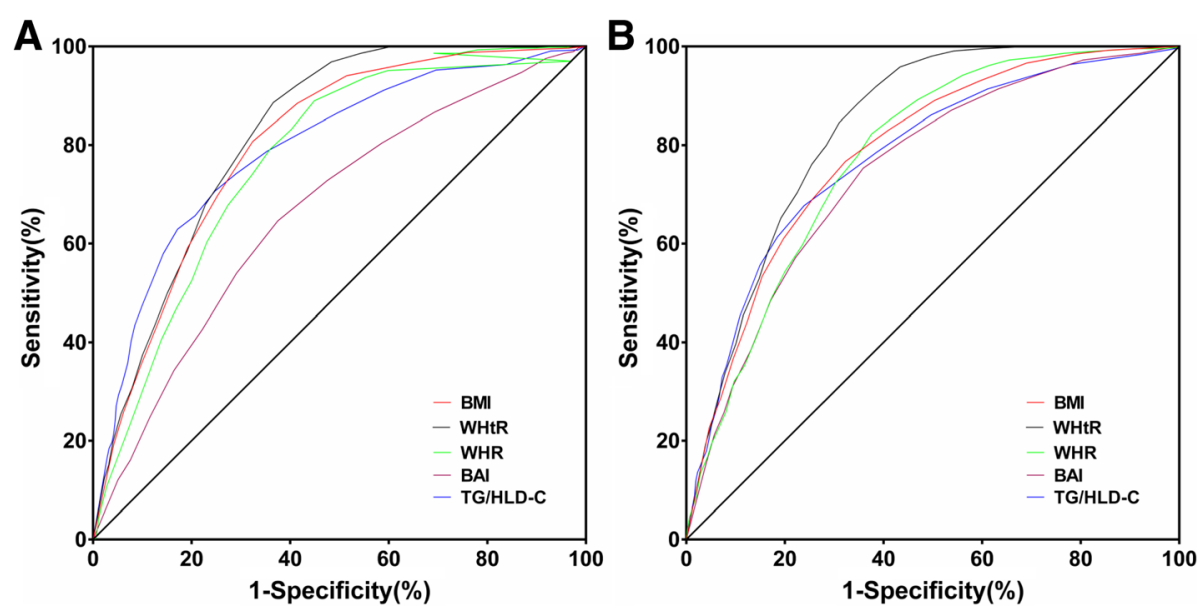

Fig. 2 ROC curves for each variable to predict the presence of MetS in Kazakh men (a) and women (b). ROC, receiver operating characteristic; MetS, metabolic syndrome; BMl, body mass index; WHtR, waist to height ratio; WHR, waist to hip ratio; BAl, body adiposity index; TG, triglycerides; HDL-C, high-density lipoprotein cholesterol

They also reported that WC had the equivalent predicting performance as the WHtR since the AUC was also 0.69. In this present study, WC was the necessary factor in the diagnosis of MetS according to IDF criteria [23], so the AUC of WC was $100 \%$. The AUC value of WHtR in our study was 0.821 , which was larger than 0.69 in study mentioned above. It suggested that WHtR may be a better indicator for MetS especially in Kazakh men, the sensitivity was $81.09 \%$ and specificity was $68.50 \%$.

In women, according to the respective ROC curves, the WHtR had the highest AUC value of 0.835 , it was followed by BMI, WHR, TG/HDL-C and BAI. Meanwhile, WHtR had the shortest distance of 0.35 in ROC (Sen $=84.59 \%$, Spe $=68.97 \%)$. The association of WHtR to MetS was followed by BMI, TG/HDL-C, WHR, and BAI according to the distance in ROC. Our results were consistent with the study by Zhang et al. [4], they reported the WHtR was best predicting indicator of MetS in Chinese women with the AUC of 0.70. Therefore, this atherogenic index may be used as the best parameter in the screening of MetS in Chinese women. Even BAI was reported to be able to reflect \%body fat of differing ethnicities without numerical correction, however, the predicting ability of BAI was the lowest in both Kazakh men and women in our study.

The cut-off values of the parameters to detect the MetS in Kazakh men were $27.00 \mathrm{~kg} / \mathrm{m}^{2}, 0.93,0.55,28.00,1.10$ for BMI, WHR, WHtR, BAI, TG/HDL, respectively. Meanwhile, the cut-off values of the parameters to detect the MetS in Kazakh women were $26.00 \mathrm{~kg} / \mathrm{m}^{2}, 0.87,0.54$, 32.00, 0.90 for BMI, WHR, WHtR, BAI, TG/HDL, respectively. BMI and WHtR values in both man and women of

Table 3 Sensitivity (Sen), specificity (Spe), distance in the receiver operating characteristic (ROC) curve and cutoffs of each variable for the presence of MetS in Kazakh men and women

\begin{tabular}{|c|c|c|c|c|c|}
\hline & Anthropometric index & Sen (\%) & Spe (\%) & Distance in ROC curve & Cutoff \\
\hline \multirow[t]{5}{*}{ Men } & BMI & 80.74 & 67.60 & 0.38 & 27.0 \\
\hline & WHR & 73.91 & 67.78 & 0.41 & 0.93 \\
\hline & WHtR & 81.09 & 68.50 & 0.37 & 0.55 \\
\hline & $\mathrm{BAl}$ & 64.62 & 62.49 & 0.52 & 28.00 \\
\hline & $\mathrm{TG} / \mathrm{HDL}-\mathrm{C}$ & 70.66 & 75.25 & 0.38 & 1.10 \\
\hline \multirow[t]{5}{*}{ Women } & BMl & 76.73 & 67.72 & 0.40 & 26.0 \\
\hline & WHR & 72.42 & 69.71 & 0.41 & 0.87 \\
\hline & WHtR & 84.59 & 68.97 & 0.35 & 0.54 \\
\hline & $\mathrm{BAl}$ & 75.35 & 64.14 & 0.44 & 32.00 \\
\hline & TG/HDL-C & 67.73 & 76.10 & 0.40 & 0.90 \\
\hline
\end{tabular}

MetS metabolic syndrome, AUC Area under the curves, BMI body mass index, WHR waist to hip ratio, WHtR waist to height ratio, $B A I$ body adiposity index, TG triglycerides, $H D L-C$ high-density lipoprotein cholesterol

The boled part means the optimal value in respect sex 
our study were higher than those in Japanese and Korean man and women $[24,25]$. We also noticed that cut-off values of BMI, WHR, WHtR and TG/HDL in Kazakh women were also higher than those in Chinese women in Guangdong [5]. The reason why the cutoffs to detect the MetS in Kazakh population were at high levels was still unclear. The ethnic difference and living habits may greatly contribute to it.

The present study has several strengths. This is the first representative sample of the general adult Kazakh population in China. Thus, these results can be generalized to the full adult Kazakh population aged above 35 years. Additionally, we provided AUC and cutoffs information for many anthropometric and atherogenic parameters stratified by sex. Future studies can use the cutoffs suggested here for the screening and intervention of MetS in a representative sample of the adult Kazakh population. The limitation of this study is its cross-sectional design, which precludes causal inferences. Second, CRS was conducted based on one time visit, the prevalence of raised blood pressure and raised fasting plasma glucose may be underestimated to some extent.

\section{Conclusion}

In this study of adult Kazakh population showed that $28.6 \%$ of men had the MetS, while $31.0 \%$ of women had the MetS in Xinjiang, China. Among all five anthropometric and atherogenic parameters, the WHtR had closest association with MetS both in men and women. This study enriched the limited data on the MetS for immediate use in screening among Kazakh population.

\section{Methods}

\section{Ethics statement}

This study was approved by the Ethics Committee of The First Affiliated Hospital of Xinjiang Medical University and was conducted according to the standards of the Declaration of Helsinki. Written, informed consent was obtained from the participants [26].

\section{Study population}

All the participants were selected from the Cardiovascular Risk Survey (CRS) study, the detailed description of the study population and the methods were described previously [27, 28]. Briefly, the CRS study used a 4-stage stratified sampling method to select a representative sample of the general population in Xinjiang, northwest of China. The research sites included Urumqi City, Kelamayi City, Fukang City, Yili Prefecture. The time period was from Oct. 2007 to Mar. 2010. The selections made from sampling units were based on geographic area, sex, and age groups using household registries. The 4-stage stratified sampling method was as follows: Stage one, according to population census data of Xinjiang in 2000, the areas mentioned above were selected based on population, ethnicity, geography, economic and cultural development level respectively. Stage two, according to the ethnic aggregation status, one district or county was randomly selected from the Kazakh population dominated area. Stage three, one community or town (village) was randomly selected from each district or county. Stage four, subjects over 35 years were randomly selected from each community or town (village) as research subjects. The staff conducted surveys in households and administered questionnaires. The questionnaires included the demographic, socioeconomic, dietary, and medical history of each participant. In total, the CRS included 14,618 participants (5757 Hans, 4767 Uighurs, and 4094 Kazakhs). Four hundred ninety-four Kazakh participants were enrolled in the present study. One thousand nine hundred ninety-nine participants were male and 2095 participants were female. The age of the participants were from 35 to 98 years old with the mean \pm SD age of $48.63 \pm 11.68$ (men, $49.25 \pm 12.03$; women, $48.03 \pm 11.31$ ).

\section{Data collection}

Data collection involved one visit in the participants' residential areas. During the examinations, a standard questionnaire assessing demographic information and medical history was collected by trained research staff. Body weight was measured while the subjects were without clothes or shoes by using a double balance placed on a firm surface. Height was measured by using a Frankfort place positioned at a $90^{\circ}$ angle against a wall-mounted metal tape. Waist and hip circumferences were measured at the level of the umbilicus, and at the level of the maximum girth between the iliac crest and the crotch, respectively. BMI was calculated as weight in $\mathrm{kg}$ divided by height in $\mathrm{m}^{2}$. WHR was calculated as the ratio of the waist-to-hip circumferences. WHtR was calculated by dividing waist circumference by height. Body adiposity index was calculated using the following equation: $\mathrm{BAI}=(($ hip circumference $\left.) /\left((\text { height })^{1.5}\right)-18\right)[3]$.

\section{Laboratory methods}

Blood samples were obtained from the antecubital vein into anticoagulant tubes containing EDTA in the morning after an overnight fasting period. All the collected samples were transported on dry ice at prearranged intervals to Xinjiang Coronary Artery Disease VIP Laboratory. The serum concentration of serum total cholesterol (TC), triglyceride (TG), low density lipoprotein (LDL), high density lipoprotein (HDL) and fasting glucose were measured by the Clinical Laboratory Department of The First Affiliated Hospital of Xinjiang Medical University with the biochemical analyzer (Dimension AR/AVL Clinical Chemistry System, Newark, NJ, USA) [29, 30]. 


\section{Blood pressure measurement}

A mercury sphygmomanometer was used to measure the blood pressure in the sitting position after a 10 min rest period. During the $30 \mathrm{~min}$ preceding the measurement, the subjects were required to refrain from smoking or consuming caffeine. The appearance of the first sound was used to define systolic blood pressure, and the disappearance of sound was used to define diastolic blood pressure [31]. Systolic and diastolic blood pressures were measured for twice, and the average of measurements was used for data analysis. If the first two measurements differed by more than $5 \mathrm{mmHg}$, additional readings were taken.

\section{Definition of metabolic syndrome}

Following the IDF criteria, metabolic syndrome was defined by central obesity (defined as waist circumference $\geq 90 \mathrm{~cm}$ for Chinese men and $\geq 80 \mathrm{~cm}$ for Chinese women, with ethnicity specific values for other groups), plus any two of the following four factors: Raised TG level: $\geq 150 \mathrm{mg} / \mathrm{dL}$ $(1.7 \mathrm{mmol} / \mathrm{L})$, or specific treatment for this lipid abnormality; Reduced HDL cholesterol: < $40 \mathrm{mg} / \mathrm{dL}(1.0 \mathrm{mmol} / \mathrm{L})$ in males and $<50 \mathrm{mg} / \mathrm{dL}(1.3 \mathrm{mmol} / \mathrm{L})$ in females, or specific treatment for this lipid abnormality; Raised blood pressure: systolic $\mathrm{BP} \geq 130$ or diastolic $\mathrm{BP} \geq 85 \mathrm{mmHg}$, or treatment of previously diagnosed hypertension; Raised fasting plasma glucose $(\mathrm{FPG}) \geq 100 \mathrm{mg} / \mathrm{dL}$ (5.6 mmol/L), or previously diagnosed type 2 diabetes; If FPG was above $5.6 \mathrm{mmol} / \mathrm{L}$ or $100 \mathrm{mg} / \mathrm{dL}$, OGTT is strongly recommended but is not necessary to define presence of the syndrome [23].

\section{Statistics}

The statistical analysis was conducted using SPSS version 17.0 for Windows (SPSS Inc., Chicago, IL, USA). Continuous variables were expressed as gender and metabolic syndrome specific means and standard deviations, and categorical variables were expressed as gender-specific proportions. Age-standardization was performed by the direct method by using the 2000 Xinjiang Kazakh population as the standard population. The area under the receiver's operating characteristic curve (AUC) and the $95 \%$ confidence intervals (CIs) were used to compare the association of various anthropometric and atherogenic parameters. Additionally, the distance on the receiver operating characteristic (ROC) curve was calculated as the square root of $\left[(1-\text { sensitivity })^{2}+(1-\text { specificity })^{2}\right]$. The parameter with the shortest distance on the ROC curve, which means the minimum distance from ROC curve to the upper left corner, was considered as the appropriate cutoff.

\section{Abbreviations}

BAI: Body adiposity index; BMI: Body mass index; DBP: Diastolic blood pressure; HDL: High-density lipoprotein cholesterol; LDL-C: Low-density lipoprotein cholesterol; MetS: Metabolic syndrome; SBP: Systolic blood pressure; TG: Triglycerides; WHR: Waist to hip ratio; WHtR: Waist to height ratio

\section{Acknowledgments}

The authors would like to thank the cardiologists, general practitioners and nurses and who collaborated on this project, as well as the participants for their cooperation during the study. Finally, we wish to thank Ori Ben-Yehuda, M.D. for providing language assistance.

\section{Funding}

This present study was supported by grants from the Xinjiang Science and Technology Support Project (Grant No. 2016E02073) and National Natural Science Foundation of China (Grant No. 81560071 and 81260048). The funders had no role in the design and analysis of the study or in the writing of this article.

\section{Availability of data and materials}

The datasets analysed during the current study are available from the corresponding author on reasonable request.

\section{Authors' contributions}

$Y M, B C, Y Y$ designed the study, $Y M, X C, C H, Y Y, F L, X M, X L$ and $X X$ performed the research, $\mathrm{CH}$ and $Y Y$ collected the data and $\mathrm{FL}$ analyzed the data, $\mathrm{CH}$ and $\mathrm{XC}$ prepared the manuscript. All authors have read and approved the final version of this manuscript.

\section{Competing interests}

The authors declare that they have no competing interests.

\section{Author details}

${ }^{1}$ Xinjiang Key Laboratory of Cardiovascular Disease, Clinical Medical Research Institute, First Affiliated Hospital of Xinjiang Medical University, Urumqi 830054, China. ${ }^{2}$ Department of Cardiology, First Affiliated Hospital of Xinjiang Medical University, No. 137 South Liyushan Road, Urumqi 830054, People's Republic of China.

Received: 18 January 2016 Accepted: 17 September 2016

Published online: 23 September 2016

\section{References}

1. Magliano DJ, Shaw JE, Zimmet PZ. How to best define the metabolic syndrome. Ann Med. 2006:38:34-41.

2. Zalesin KC, Franklin BA, Miller WM, Peterson ED, McCullough PA. Impact of obesity on cardiovascular disease. Med Clin North Am. 2011;95:919-37.

3. Bergman RN, Stefanovski D, Buchanan TA, Sumner AE, Reynolds JC, Sebring NG, Xiang AH, Watanabe RM. A better index of body adiposity. Obesity (Silver Spring). 2011;19:1083-9.

4. Zhang ZQ, Deng J, He LP, Ling WH, Su YX, Chen YM. Comparison of various anthropometric and body fat indices in identifying cardiometabolic disturbances in Chinese men and women. PLOS One. 2013:8:e70893.

5. Liang H, Chen X, Chen Q, Wang Y, Wu X, Li Y, Pan B, Liu H, Li M. The metabolic syndrome among postmenopausal women in rural Canton: prevalence, associated factors, and the optimal obesity and atherogenic indices. PLoS One. 2013;8:e74121.

6. Cai L, Liu A, Zhang Y, Wang P. Waist-to-height ratio and cardiovascular risk factors among Chinese adults in Beijing. PLoS One. 2013;8:e69298.

7. Gu D, Reynolds K, Wu X, Chen J, Duan X, Reynolds RF, Whelton PK, He J. Prevalence of the metabolic syndrome and overweight among adults in China. Lancet. 2005;365:1398-405.

8. Motala AA, Esterhuizen T, Pirie FJ, Omar MA. The prevalence of metabolic syndrome and determination of the optimal waist circumference cutoff points in a rural South african community. Diabetes Care. 2011:34:1032-7.

9. Khanam MA, Qiu C, Lindeboom W, Streatfield PK, Kabir ZN, Wahlin A. The metabolic syndrome: prevalence, associated factors, and impact on survival among older persons in rural Bangladesh. PLoS One. 2011;6:e20259.

10. Ding QF, Hayashi T, Zhang XJ, Funami J, Ge L, Li J, Huang XL, Cao L, Zhang J, Akihisa I. Risks of CHD identified by different criteria of metabolic syndrome and related changes of adipocytokines in elderly postmenopausal women. J Diabet Complications. 2007:21:315-9.

11. Kim HM, Park J, Ryu SY, Kim J. The effect of menopause on the metabolic syndrome among Korean women: the Korean National Health and Nutrition Examination Survey, 2001. Diabetes Care. 2007;30:701-6.

12. Cho GJ, Lee JH, Park HT, Shin JH, Hong SC, Kim T, Hur JY, Lee KW, Park YK, $\mathrm{Kim} \mathrm{SH}$. Postmenopausal status according to years since menopause as 
an independent risk factor for the metabolic syndrome. Menopause. 2008;15:524-9.

13. Lao XQ, Zhang YH, Wong MC, Xu YJ, Xu HF, Nie SP, Ma WJ, Thomas GN, Yu IT. The prevalence of metabolic syndrome and cardiovascular risk factors in adults in southern China. BMC Public Health. 2012;12:64.

14. Lao XQ, Thomas GN, Jiang CQ, Zhang WS, Yin P, Adab P, Lam TH, Cheng KK. Association of the metabolic syndrome with vascular disease in an older Chinese population: Guangzhou Biobank Cohort Study. J Endocrinol Invest. 2006;29:989-96

15. Muller MJ, Lagerpusch M, Enderle J, Schautz B, Heller M, Bosy-Westphal A. Beyond the body mass index: tracking body composition in the pathogenesis of obesity and the metabolic syndrome. Obes Rev. 2012;13 Suppl 2:6-13.

16. Feng Y, Hong X, Li Z, Zhang W, Jin D, Liu X, Zhang Y, Hu FB, Wei LJ, Zang T, et al. Prevalence of metabolic syndrome and its relation to body composition in a Chinese rural population. Obesity (Silver Spring). 2006;14:2089-98.

17. Cai H, Huang J, Xu G, Yang Z, Liu M, Mi Y, Liu W, Wang H, Qian D. Prevalence and determinants of metabolic syndrome among women in Chinese rural areas. PLoS One. 2012;7:e36936.

18. Fakhrzadeh H, Ebrahimpour P, Pourebrahim R, Heshmat R, Larijani B. Metabolic Syndrome and its Associated Risk Factors in Healthy Adults: APopulation-Based Study in Iran. Metab Syndr Relat Disord. 2006:4:28-34.

19. Zuo H, Shi Z, Hu X, Wu M, Guo Z, Hussain A. Prevalence of metabolic syndrome and factors associated with its components in Chinese adults. Metabolism. 2009;58:1102-8.

20. Wildman RP, Gu D, Reynolds K, Duan X, He J. Appropriate body mass index and waist circumference cutoffs for categorization of overweight and central adiposity among Chinese adults. Am J Clin Nutr. 2004;80:1129-36.

21. Paajanen TA, Oksala NK, Kuukasjarvi P, Karhunen PJ. Short stature is associated with coronary heart disease: a systematic review of the literature and a meta-analysis. Eur Heart J. 2010;31:1802-9.

22. Henriksson KM, Lindblad U, Agren B, Nilsson-Ehle P, Rastam L. Associations between body height, body composition and cholesterol levels in middleaged men. the coronary risk factor study in southern Sweden (CRISS). Eur J Epidemiol. 2001;17:521-6.

23. Alberti KG, Zimmet $P$, Shaw J. The metabolic syndrome-a new worldwide definition. Lancet. 2005:366:1059-62.

24. Nakamura K, Nanri H, Hara M, Higaki Y, Imaizumi T, Taguchi N, Sakamoto T, Horita M, Shinchi K, Tanaka K. Optimal cutoff values of waist circumference and the discriminatory performance of other anthropometric indices to detect the clustering of cardiovascular risk factors for metabolic syndrome in Japanese men and women. Environ Health Prev Med. 2011;16:52-60.

25. Ko KP, Oh DK, Min H, Kim CS, Park JK, Kim Y, Kim SS. Prospective study of optimal obesity index cutoffs for predicting development of multiple metabolic risk factors: the Korean genome and epidemiology study. J Epidemiol. 2012;22:433-9.

26. Chen BD, He CH, Ma YT, Yang YN, Liu F, Pan S, Ma X, Li XM, Fu ZY, Xie X, Zheng YY. Best anthropometric and atherogenic predictors of metabolic syndrome in the Chinese Han population in Xinjiang: the Cardiovascular Risk Survey. Ann Nutr Metab. 2014:65:280-8.

27. Pan S, Yu ZX, Ma YT, Liu F, Yang YN, Ma X, Fu ZY, Li XM, Xie X, Chen Y, et al. Appropriate body mass index and waist circumference cutoffs for categorization of overweight and central adiposity among Uighur adults in Xinjiang. PLoS One. 2013;8:e80185.

28. He CH, Pan S, Ma YT, Yang YN, Ma X, Li XM, Xie X, Chen Y, Yu ZX, Chen BD, et al. Optimal waist-to-height ratio cutoff values for predicting cardio-metabolic risk in Han and Uygur adults in northwest part of China. Eur J Clin Nutr. 2015;69:954-60

29. Pan S, He CH, Ma YT, Yang YN, Ma X, Fu ZY, Li XM, Xie X, Yu ZX, Chen Y, et al. Serum uric acid levels are associated with high blood pressure in Chinese children and adolescents aged 10-15 years. J Hypertens. 2014:32:998-1004.

30. Xie X, Ma YT, Yang YN, Fu ZY, Li XM, Zheng YY, Huang D, Ma X, Chen BD, Liu F. Polymorphisms in the SAA1 gene are associated with ankle-to-brachial index in Han Chinese healthy subjects. Blood Press. 2011;20:232-8.

31. American Society of Hypertension. Recommendations for routine blood pressure measurement by indirect cuff sphygmomanometry. American Society of Hypertension. Am J Hypertens. 1992;5:207-9. 